\title{
WiFi concierge at home network focusing on streaming traffic
}

\author{
Satoru Nishimaki ${ }^{a)}$, Hiroshi Yamamoto, and Katsuyuki Yamazaki \\ Nagaoka University of Technology, \\ 1603-1 Kamitomioka, Nagaoka, Niigata 940-2188, Japan \\ a)s_nishimaki@stn.nagaokaut.ac.jp
}

\begin{abstract}
Channel interference in wireless local area networks (LANs) has become an unavoidable problem in home networks (HNWs) with the widespread use of smartphones and tablets. In addition, streaming video over WiFi has become popular on HNWs. However, channel interference in streaming traffic continues longer than that in other data traffic and deteriorates the quality of communications of all other devices using the same channel. Therefore, this paper proposes a new WiFi concierge system that visualizes the conditions of WiFi channels by using new methods of estimating channel congestion and detecting streaming traffic to avoid channels where interference has occurred due to streaming traffic. Furthermore, monitoring experiments were conducted in several apartments to test and investigate the validity of the proposed system. As a result, we concluded that the proposed system could recommend better channels to its users.
\end{abstract}

Keywords: wireless LAN, channel interference, home network, streaming traffic, congestion estimation

Classification: Wireless Communication Technologies

\section{References}

[1] A. P. Jardosh, K. N. Ramachandran, K. C. Almeroth, and E. M. Belding-Royer, "Understanding congestion in IEEE 802.11b wireless networks," Proc. of the 2005 Internet Measurement Conference, pp. 279-292, 2005.

[2] J. Choi, K. Lee, S. R. Lee, and J. Ihm, "Channel selection for IEEE 802.11 based wireless LANs using $2.4 \mathrm{GHz}$ band," IEICE Electron. Express, vol. 8, no. 16, pp. 1275-1280, 2011. DOI:10.1587/elex.8.1275

[3] S. Nishimaki, H. Yamamoto, and K. Yamazaki, "Detection and characteristic analysis of streaming traffic on wireless LAN," Proc. of the 2014 IEICE General Conference, ISS-SP-370 (in Japanese).

[4] T. Yutaka, S. Yoh, and T. Osamu, "Optimal channel selection in $2.4 \mathrm{GHz}$ band using duty cycle," IEICE Technical Report, pp. 193-198, October 2010.

[5] U. Paul, A. Kashyap, R. Maheshwari, and S. R. Das, "Passive measurement of interference in WiFi networks with application in misbehavior detection," IEEE Trans. Mobile Comput., vol. 12, no. 3, pp. 434-446, March 2013. DOI:10. 1109/TMC.2011.259

[6] B. A. Hirantha, K. Ishihara, Y. Inoue, T. Ichikawa, T. Kumagai, and M. Mizoguchi, "Proposal of master-slave channel selection scheme for IEEE 802.11-based wireless LAN systems," Proc. of 2012 IEICE General Conference, 
p. 520, March 2013.

[7] K. Ohno, R. Suto, and R. S. Atarashi, “A study on network concierge," IEICE Technical Reports, pp. 13-20, July 2008.

\section{Introduction}

Many wireless devices (e.g., smart phones, tablets, and laptops) have been used in recent years in home networks (HNWs). As a result, channel interference in wireless local area networks (LANs) has become an unavoidable problem especially when many people are living in limited areas (e.g., apartment houses, and densely populated residential areas). This is because there are only three channels that can be allocated to wireless LANs without creating any interference in the $2.4 \mathrm{GHz}$ frequency band. Channel interference degrades the performance of communication $[1,2]$. In addition, video streaming over WiFi (e.g., the Digital Living Network Alliance (DLNA)) has become popular on HNWs. Channel interference in streaming traffic continues longer than that in other data traffic (e.g., that from Web browsing), which deteriorates the communications quality of all devices using the same channel of wireless LANs [3]. A tool measuring the Received Signal Strength Indication (RSSI) of each channel has been proposed to solve this problem. However, it is impossible for the existing tool to estimate what influence channel interference has on communications quality or to detect streaming traffic only based on RSSI.

Therefore, we propose a new WiFi concierge system in this paper to help non-IT people to understand the state of wireless channels and to select appropriate settings for the access point (AP). The system is designed to monitor the state of wireless channels 24 hours a day and 365 days a year to estimate the communications quality in individual WiFi channels, and to detect devices generating streaming traffic by utilizing a wireless LAN adapter configured as the monitor mode. Furthermore, the proposed system is implemented as a WiFi concierge system that provides the WiFi channel state for non-IT people, and provides comprehensive guides to configure their APs. This information is displayed on a TV screen.

\section{Related work}

\subsection{Estimates of wireless network quality in home networks}

Channel assignment based on RSSI has been studied [4] as a technology to avoid channel interference. RSSI can be detected with an existing tool (e.g., inSSIDer), and the user can select a suitable channel where the total RSSI of APs is the smallest. However, it is impossible for the tool to exactly estimate communications quality and to detect streaming traffic only based on RSSI. Furthermore, it is hard for non-IT people to install and use the tool.

In addition, a method of estimating channel congestion based on monitored WiFi packets has been studied [5]. However, these studies have only targeted one channel, and calculated the usage rate of communications media in the medium access control (MAC) layer of wireless LANs. Therefore, a system that measures 
network performance metrics directly related to application quality (e.g., packet loss rate and round-trip time (RTT)) in all channels is necessary for HNW users to be able to select the best channel.

\subsection{Channel setting function}

As it is difficult for non-IT people to configure WiFi APs, an auto channel setting function has been implemented in commercially available APs. The function automatically selects unused channels based on surrounding wireless LAN conditions. However, it evaluates the condition of individual channels only based on RSSI, but does not consider the amount of real traffic. In addition, the configuration is done only once during the power-on sequence of the AP [6]. Therefore, it cannot respond to dynamic changes under wireless network conditions. Furthermore, existing systems have not been designed to enable non-IT people to easily understand how to change WiFi settings.

Concierge systems are also popular as supporting systems that provide suitable suggestions based on knowledge and situations [7]. A concierge system that reports channel conditions and supports the changing of channel settings so that even nonIT people can understand them is needed in HNWs for these people to easily utilize wireless LANs.

\section{Overview of proposed system}

We propose a new WiFi concierge system for non-IT people that focuses on estimating congestion and detecting streaming traffic in individual WiFi channels that takes into considerations the situations that were explained in Section 2. Fig. 1(a) overviews the proposed WiFi concierge system. The main component of our proposed system is a single-board monitor device (BeagleBone Black), which is equipped with a wireless LAN adapter. A television is used as an information display to enable non-IT people to easily use the system.

The wireless LAN adapter is configured in the monitor mode to monitor data traffic in individual wireless channels and monitor the headers of data frames on surrounding wireless LANs without associating with any WiFi APs. The contents of the data frames are encrypted, but various information (e.g., frame sizes and retry flags) can be obtained from the header. The proposed system estimates communications quality, and detects streaming traffic by analyzing this information. Fur-

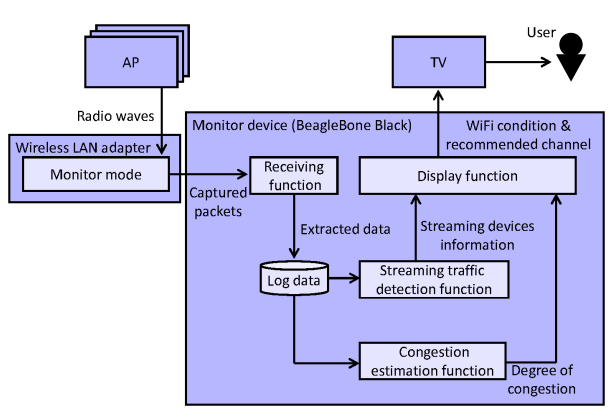

(a) Block diagram of system

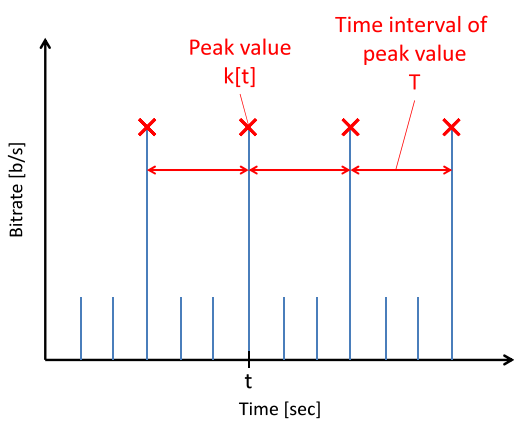

(b) Peak value detection

Fig. 1. Overview of proposed system. 
thermore, the system indicates the utilization conditions of individual WiFi channels, and recommends the best channels to the users. Based on the information on the TV, HNW users can confirm wireless LAN conditions and can select better channels for their APs.

\section{Proposed method of measuring quality of WiFi channels}

We analyzed the real data traffic of a wireless LAN measured with a wireless LAN adapter to design a method of estimating the communications quality in individual wireless LAN channels and of detecting streaming traffic. As a result of a preliminary evaluation, channel congestion in the wireless LAN was effectively estimated by measuring the retry ratios of the WiFi frames, and streaming traffic could be detected by sensing specific variations in the bitrates. Therefore, the new methods were proposed based on the characteristic patterns of WiFi traffic.

\subsection{Method of estimating congestion}

The retry ratio remains within $10[\%]$ when the bitrate is smaller than $25[\mathrm{Mb} / \mathrm{s}]$ from an analysis of real traffic obtained from the wireless LAN. However, the retry ratio markedly increased from $10[\%]$ as the bitrate increased by exceeding 25 $[\mathrm{Mb} / \mathrm{s}]$. Therefore, the system determined that the wireless channels had been transferred to an abnormal state when the retry ratio became more than $10[\%]$. The system attempted to identify devices generating streaming traffic in the abnormal state. If streaming devices were found at users' homes, the system displayed a list of the streaming devices. The WiFi concierge function of the system recommended that users change wireless channels so that network congestion could be avoided if streaming traffic was generated by devices outside the home. Here, the system evaluated the retry ratios of other channels, and displayed the best channel. If no streaming traffic could be found, the system notified HNW users of the fact that wireless conditions were deteriorating due to network congestion.

\subsection{Method of detecting streaming traffic}

We found that streaming traffic periodically generated high bitrates by analyzing streaming traffic over WiFi. Therefore, the system detected streaming traffic by analyzing the time intervals between peak values of high bitrates, as plotted in Fig. 1(b). Here, $k[t][\mathrm{b} / \mathrm{s}]$ is a one-second average bitrate when the time is $t[\mathrm{sec}]$. The method of detection treats $k[t]$ as a peak value when $k[t]$ satisfies the following two conditions:

$$
\begin{aligned}
& k[t-1]+\sigma<k[t] \\
& k[t]>k[t+1]+\sigma .
\end{aligned}
$$

Here, $\sigma[\mathrm{b} / \mathrm{s}]$ is the threshold for the bitrate. We set $20[\mathrm{~kb} / \mathrm{s}]$ to $\sigma$ in this study. In addition, time interval $T_{i}$ between two successive ( $i$-th and $i+1$-th) peaks was calculated, and streaming traffic detection utilized variance in $T_{i}$. The method was processed in three steps:

1. The bitrate of data traffic between each pair of the destination MAC address and the source MAC address is calculated, and the peak values of the bitrate are derived based on Eqs. (1) and (2). 
2. The time interval of successive peak values and variance in the intervals are calculated.

3. A streaming device is detected when the variance in the time intervals is lower than $\rho$.

Here, $\rho$ is the threshold for variance, and 25 is set to $\rho$. The method is applied to monitored data traffic every $5 \mathrm{~min}$.

\section{Experimental evaluation in home networks}

The proposed WiFi concierge system was developed to investigate its feasibility, and the monitoring experiments were carried out in several apartments where neighboring rooms were occupied. Many APs were found by using inSSIDer at the experimental location. The experimental period in each apartment was about a week.

\subsection{Experiment-1: Identified non-congested channel}

Fig. 2(a) plots the estimated results for the degree of congestion (retry ratios) in individual channels, where the degree of congestion on weekends is higher than that on weekdays. The time changes in the degree of congestion reflect the lifestyles of HNW users. Therefore, it could be concluded that the proposed system could capture actual time changes under surrounding wireless LAN conditions. In addition, Fig. 2(b) plots the average degree of congestion for the experimental period, where we can see $6 \mathrm{ch}$ has been selected by the AP, but $1 \mathrm{ch}$ has been estimated to be a better channel by the proposed system. In this case, the system can recommend a better channel for users to select based on the results obtained from estimates.

\subsection{Experiment-2: Identified streaming traffic}

After the first experiment, we evaluated the effectiveness of the proposed method of detecting streaming traffic in terms of recall, precision, and the F-measure. The results obtained from evaluating the proposed method of detection when popular video streaming services and applications were used are summarized in Table I. With all streaming services as a target, we can see recall has achieved a high value of $75.0[\%]$, precision has achieved a high value of $90.0[\%]$, and the F-measure has achieved a high value of $81.8[\%]$. These results suggest that the proposed method

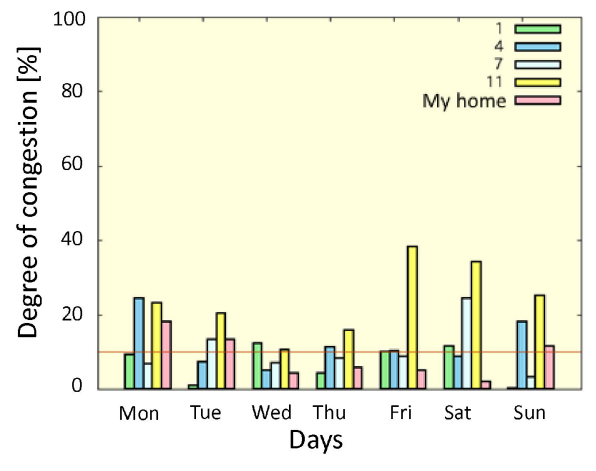

(a) Each day

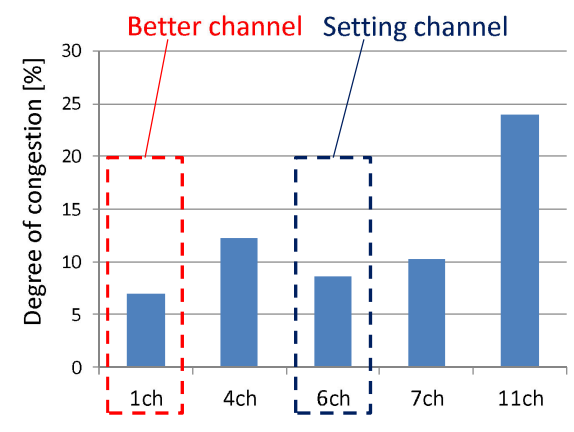

(b) Average for experimental period

Fig. 2. Network conditions per day and their average. 
Table I. Results from detecting streaming traffic

\begin{tabular}{|c|c|c|c|c|}
\hline $\begin{array}{c}\text { Types of } \\
\text { Streaming traffic }\end{array}$ & Applications & $\begin{array}{c}\text { Recall } \\
{[\%]}\end{array}$ & $\begin{array}{c}\text { Precision } \\
{[\%]}\end{array}$ & $\begin{array}{c}\text { F-measure } \\
{[\%]}\end{array}$ \\
\hline \multirow{2}{*}{ Streaming video } & Xperia movie app & 66.7 & & \\
\cline { 2 - 3 } & WMP & 83.3 & \multirow{2}{*}{-} & - \\
\hline \multirow{2}{*}{ Live broadcasting } & Ustream & 83.3 & & \\
\cline { 2 - 3 } & nicovideo & 66.7 & & \\
\hline \multicolumn{2}{|c|}{ All streaming services } & 75.0 & 90.0 & 81.8 \\
\hline
\end{tabular}

can accurately detect video streaming, and it can recommend channels where interference does not occur due to streaming traffic.

\section{Conclusion and future work}

This paper proposed a new WiFi concierge system that detected the occurrence of channel interference due to streaming traffic and alerted HNW users. As a result of a preliminary evaluation, channel congestion in a wireless LAN was effectively estimated by measuring the retry ratios of WiFi frames, and streaming traffic could be detected by detecting specific variations in bitrates. Therefore, the new methods were proposed based on the characteristic patterns of WiFi traffic. Furthermore, the proposed system was developed by using a single-board monitoring device equipped with a wireless LAN adapter. We found that the system could monitor time changes in channel congestion based on the retry ratios through experimental evaluations, and time changes in the degree of congestion reflected the lifestyles of HNW users. In addition, the detection of streaming traffic achieved high recall, precision, and the F-measure that corresponded to 75.0[\%], 90.0[\%], and 81.8[\%]. We intend to develop an auto configuration function in future work that utilizes the degree of congestion and detects streaming traffic. This study was partly supported by the KDDI Foundation, Japan. 